\title{
The effect of spin-orbit interaction on entanglement of two-qubit Heisenberg XYZ systems in an inhomogeneous magnetic field
}

\author{
Fardin Kheirandish*, S. Javad Akhtarshenas ${ }^{\dagger}$ and Hamidreza Mohammadi $\ddagger$ \\ Department of Physics, University of Isfahan, Hezar Jarib Ave., Isfahan, Iran
}

\begin{abstract}
The role of spin-orbit interaction on the ground state and thermal entanglement of a Heisenberg XYZ two-qubit system in the presence of an inhomogeneous magnetic field is investigated. For a certain value of spin-orbit parameter $D$, the ground state entanglement tends to vanish suddenly and when $D$ crosses its critical value $D_{c}$, the entanglement undergoes a revival. The maximum value of the entanglement occurs in the revival region. In the finite temperatures, there are revival regions in $D-T$ plane where increasing of temperature first increases the entanglement and then tends to decrease it and ultimately vanishes for temperatures above a critical temperature. This critical temperature is an increasing function of $D$, thus the nonzero entanglement can exist for larger temperatures. In addition, the amount of entanglement in the revival region depends on the spin-orbit parameter. Also, entanglement teleportation via the quantum channel constructing by above system is investigated and influence of spin-orbit interaction on the fidelity of teleportation and entanglement of replica state is studied.
\end{abstract}

PACS numbers: 03.67.Hk, 03.65.Ud, 75.10.Jm

\section{INTRODUCTION}

A. Einstein, B. Podolsky and N. Rosen, in their famous EPR paradox, argued that in general two quantum system cannot be separated even if they are located far from each other [1]. E. Schrödinger named this quantum mechanical property as Entanglement [2]. Today, entanglement is a uniquely quantum mechanical resource that plays a key role in many of the most interesting applications of quantum computation and quantum information $[3,4]$. Thus a great deal of efforts have been devoted to study and characterize the entanglement in the recent years. The central task of quantum information theory is to characterize and quantify entanglement of a given system. A mixed state $\rho$ of a bipartite system is said to be separable or classically correlated if it can be expressed as a convex combination of uncorrelated states $\rho_{A}$ and $\rho_{B}$ of each subsystems i.e. $\rho=\sum_{i} \omega_{i} \rho_{A}^{i} \otimes \rho_{B}^{i}$ such that $\omega_{i} \geq 0$ and $\sum_{i} \omega_{i}=1$, otherwise $\rho$ is entangled [4, 5]. Many measures of entanglement have been introduced and analyzed [3, 6, 7], but the one most relevant to this work is entanglement of formation, which is intended to quantify the resources need to create a given entangled state [6]. For the case of a two-qubit system Wootters has shown that entanglement of formation can be obtained explicitly as:

$$
E(\rho)=\Xi[C(\rho)]=h\left(\frac{1+\sqrt{1+C^{2}}}{2}\right),
$$

\footnotetext{
${ }^{*}$ fardin_kh@phys.ui.ac.ir

†akhtarshenas@phys.ui.ac.ir

${ }^{\ddagger}$ h.mohammadi@phys.ui.ac.ir
}

where $h(x)=-x \log _{2} x-(1-x) \log _{2}(1-x)$ is the binary entropy function and $C(\rho)=\max \left\{0,2 \lambda_{\max }-\sum_{i=1}^{4} \lambda_{i}\right\}$ is the concurrence of the state, where $\lambda_{i}$ s are positive square roots of the eigenvalues of the non-Hermitian ma$\operatorname{trix} R=\rho \tilde{\rho}$, and $\tilde{\rho}$ is defined by $\tilde{\rho}:=\left(\sigma^{y} \otimes \sigma^{y}\right) \rho^{*}\left(\sigma^{y} \otimes \sigma^{y}\right)$. The function $\Xi$ is a monotonically increasing function and ranges from 0 to 1 as $\mathrm{C}$ goes from 0 to 1 , so that one can take the concurrence as a measure of entanglement in its own right. In the case that the state of the system is pure i.e. $\rho=|\psi\rangle\langle\psi|,| \psi\rangle=a|00\rangle+b|01\rangle+c|10\rangle+d|11\rangle$, the above formula is simplified to $C(|\psi\rangle)=2|a d-b c|$. The spin chain is the natural candidates for the realization of entanglement and Heisenberg model is the simplest method for studying and investigating the behavior of the spin chains . Nielsen [8] is the first person who studied the entanglement of two-qubit Heisenberg XXX- chain modeled with the Hamiltonian $H=$ $J \boldsymbol{\sigma}_{\mathbf{1}} \cdot \boldsymbol{\sigma}_{\mathbf{2}}+\mathbf{B} \cdot\left(\sigma_{1}^{z}+\sigma_{2}^{z}\right)$. He showed that entanglement in such systems exists only for antiferromagnatic $(J>0)$ case below a threshold temperature $T_{c}$. After Nielsen, entanglement in the two-qubit XXX, XXZ and XY systems in the presence of homogeneous and inhomogeneous magnetic field has been investigated [9, 10, 11, 12, 13, 14]. The effect of anisotropy due to spin coupling in the $\mathrm{x}, \mathrm{y}, \mathrm{z}$ direction has also studied in a number of works [15, 16]. In ref [17] Yang et al. have shown that in XYZ Heisenberg systems, an inhomogeneous external magnetic field can make the larger revival, improve the critical temperature and enhance the entanglement. Spinorbit (SO) interaction cause another type of anisotropy 18, 19, 20, 21, 22, 23, 24]. The effect of SO interaction in the thermal entanglement of a two-qubit XXX system in the absence of magnetic field has been studied in [25]. However, the entanglement for a XYZ Heisenberg system under an inhomogeneous magnetic field in the presence of SO interaction has not been discussed. Therefore, in this paper we investigate the influence of SO interaction 
on the entanglement and entanglement teleportation of two-qubit system at thermal equilibrium.

On the other hand, among the numerous concepts to implement a quantum bit (qubit), approaches based on semiconductor Quantum Dots (QDs) offer the great advantage that ultimately a miniaturized version of quantum computer is feasible. Indeed, at first D. Loss and D.P. Diviencenzo proposed a quantum computer protocol based on electron spin trapped in semiconductor QDs in 1998 [26, 27, 28]. Here, the qubit is represented by a single electron in a QD which can initialize, manipulate and read out by extremely sensitive devices. Compared with other systems such as quantum optical systems [29] and nuclear magnetic resonance (NMR) 30, 31, 32], QDs are argued to be more scalable and has long decoherence time [3]. The above Heisenberg system is suitable for modeling and computing the entanglement of a two-qubit system represented by two electrons confined in two vertically coupled quantum dots (CQDs), respectively. Due to weak lateral confinement electrons can tunnel from one dot to another dot and spin-spin and spin-orbit interaction between the two qubits exists. In GaAs double QDs, hyperfine interactions have been identified to dominate spin mixing at small magnetic fields, while SO interaction is not relevant in this regime. However SO interaction and the coupling magnetic fields are expected to be orders of magnitude stronger in InAs compared to GaAs [33. SO interaction in such nanostructures can be investigated with the help of quantum optical methods [34].

Taking the advantage of tunability of SO strength [34, 35, 36, 37], we show that this type of interaction cause to enhancement of entanglement in the revival region, increase the volume of revival region and improve the critical values of other parameters.

In the following, as an application of the above system, the entanglement teleportation of a two-qubit pure state via the above two-qubit system is investigated and average fidelity between input and output states is calculated. C. Bennet et al. have shown that two entangled spatially separated particles can be used for teleportation [38]. They also argued that states which are less entangled still could be used for teleportation but they reduce "the fidelity of teleportation and/or the range of state $|\phi\rangle$ which can accurately be teleported". After then S. Popscu by using hidden variable model, have shown that, teleportation of a quantum state via a pure classical communication cannot performed with fidelity larger than $\frac{2}{3}$ [39]. Thus, mixed quantum channels which allows to transfer the quantum information with the fidelity larger than $\frac{2}{3}$ are worthwhile. Horodecki et al. have calculated the optimal fidelity of teleportation for bipartite state acting on $C^{d} \otimes C^{d}$ by using the isomorphism between quantum channels and a class of bipartite states and twirling operations [40]. G. Bowen and S. Bose have shown that "standard teleportation with an arbitrary mixed state resource is equivalent to general depolarizing channel with the probabilities given by the maximally entangled component of the resource. This enables the usage of any quantum channel as a generalized depolarizing channel without additional twirling operation" [41]. Using the property of linearity of teleportation process [38], J. Lee and M.S. Kim have shown that "quantum teleportation preserves the nature of quantum correlation in the unknown entangled state if the channel is quantum mechanically correlated" and then they investigate entanglement teleportation via two copies of werner states 42]. The entanglement teleportation via thermally entangled state of a two-qubit Heisenberg XXchain and XY-chain has been studied by Ye Yeo et al. [43, 44]. The effect of spin-orbit interaction on entanglement teleportation on a two-qubit XXX-Heisenberg chain in the absence of magnetic field is reported by G. Zhang [45].

In this paper we investigate the ability of the above mentioned two-qubit system for the entanglement teleportation. We show that spin-orbit interaction and inhomogeneous magnetic field have effective effect on the entanglement of replica state and the fidelity of teleportation. A minimal entanglement of the thermal state in the model is required to realize efficient entanglement teleportation and we can attain to this minimal entanglement, in the case of $J_{z}<0$, by introducing $\mathrm{SO}$ interaction.

The paper is organized as follows. In sec. 2 we introduce the Hamiltonian of a two qubit Heisenberg system under inhomogeneous magnetic field with SO interaction and write the thermal density matrix of the system related to this Hamiltonian and ultimately calculate the thermal concurrence of the system. In subsection 2.1, the ground state entanglement is calculated and results are plotted in figs. 1-2c. In subsection 2.2 the finite temperature entanglement of system is computed. The figs. 3-5 illustrate the obtained results. The entanglement teleportation of a two-qubit pure state and its fidelity derived in sec. 3 and results are plotted in figs. 6-8. In sec. 4 a discussion concludes the paper.

\section{MODEL AND HAMILTONIAN}

The Hamiltonian of a two-qubit anisotropic Heisenberg XYZ-model in the presence of inhomogeneous magnetic field and spin-orbit interaction is:

$$
\begin{aligned}
H & =\frac{1}{2}\left(J_{x} \sigma_{1}^{x} \sigma_{2}^{x}+J_{y} \sigma_{1}^{y} \sigma_{2}^{y}+J_{z} \sigma_{1}^{z} \sigma_{2}^{z}+\mathbf{B}_{1} \cdot \boldsymbol{\sigma}_{1}+\mathbf{B}_{2} \cdot \boldsymbol{\sigma}_{2}\right. \\
& \left.+\mathbf{D} \cdot\left(\boldsymbol{\sigma}_{1} \times \boldsymbol{\sigma}_{2}\right)+\delta \boldsymbol{\sigma}_{1} \cdot \overline{\boldsymbol{\Gamma}} \cdot \boldsymbol{\sigma}_{2}\right),
\end{aligned}
$$

where $\boldsymbol{\sigma}_{j}=\left(\sigma_{j}^{x}, \sigma_{j}^{y}, \sigma_{j}^{z}\right)$ is the vector of Pauli matrices, $\mathbf{B}_{j}(j=1,2)$ is the magnetic field on site $\mathbf{j}$, $J_{\mu}(\mu=x, y, z)$ are the real coupling coefficients (the chain is anti-ferromagnetic (AFM) for $J_{\mu}>0$ and ferromagnetic $(\mathrm{FM})$ for $\left.J_{\mu}<0\right)$ and $\mathbf{D}$ is called DzyaloshinskiMoriya vector, which is first order in spin-orbit coupling and is proportional to the coupling coefficients $\left(J_{\mu}\right)$ and $\overline{\boldsymbol{\Gamma}}$ is symmetric tensor which is second order in spinorbit coupling [18, 19, 20, 21]. For simplicity we assume 
$\mathbf{B}_{j}=B_{j} \hat{z}$ such that $B_{1}=B+b$ and $B_{2}=B-b$, where b indicates the amount of inhomogeneity of magnetic field. The vector $\mathbf{D}$ and the parameter $\delta$ are dimensionless, in system like coupled GaAs quantum dots $|\mathbf{D}|$ is of order of a few percent, while the order of last term is $10^{-4}$ and is negligible. If $\mathbf{D}=J_{z} D \hat{\boldsymbol{z}}$ and ignore the second order spin-orbit coupling, then the above Hamiltonian can be expressed as:
In the above equations $N^{ \pm}=\frac{1}{\sqrt{1+\frac{(b \pm \xi)^{2}}{J^{2}+\left(J_{z} D\right)^{2}}}}$ and $M^{ \pm}=$ $\frac{1}{\sqrt{1+\left(\frac{B \pm \eta}{J \gamma}\right)^{2}}}$ are the normalization constants. Here we define, $\xi:=\sqrt{b^{2}+J^{2}+\left(J_{z} D\right)^{2}}$ and $\eta:=\sqrt{B^{2}+(J \gamma)^{2}}$, for later convenience.

The state (density matrix) of a system in equilibrium at temperature $\mathrm{T}$ is $\rho=Z^{-1} \exp \left(-\frac{H}{k_{B} T}\right)$, where $\mathrm{Z}$ is the partition function of the system and $k_{B}$ is the Boltzman constant. For simplicity we take $k_{B}=1$. In the standard $\sigma_{\mathrm{p}}^{-} \mathrm{xs}_{\mathrm{s} \mathrm{s}}^{+}$, the density matrix of the system in the thermal equilibrium can be written as:

$$
\rho_{T}=\left(\begin{array}{cccc}
\mu_{+} & 0 & 0 & \nu \\
0 & w_{1} & z & 0 \\
0 & z^{*} & w_{2} & 0 \\
\nu & 0 & 0 & \mu_{-}
\end{array}\right)
$$

of anisotropy in the XY-plane (partial anisotropy, $-1 \leq \gamma \leq 1)$ and $\sigma^{ \pm}=\frac{1}{2}\left(\sigma^{x} \pm \sigma^{y}\right)$ are lowering and raising operators.

The Hamiltonian (3), in the standard basis $\{|00\rangle,|01\rangle,|10\rangle,|11\rangle\}$, has the following matrix form:

$$
H=\left(\begin{array}{cccc}
\frac{1}{2} J_{z}+B & 0 & 0 & J \gamma \\
0 & -\frac{1}{2} J_{z}+b & J+i J_{z} D & 0 \\
0 & J-i J_{z} D & -\frac{1}{2} J_{z}-b & 0 \\
J \gamma & 0 & 0 & \frac{1}{2} J_{z}-B
\end{array}\right)
$$

The spectum of $\mathrm{H}$ is easily obtained as

$$
\begin{aligned}
& H\left|\psi^{ \pm}\right\rangle=\varepsilon_{1,2}\left|\psi^{ \pm}\right\rangle, \\
& H\left|\Sigma^{ \pm}\right\rangle=\varepsilon_{3,4}\left|\Sigma^{ \pm}\right\rangle,
\end{aligned}
$$

where the eigenstates and the corresponding eigenvalues are, respectively

$$
\begin{aligned}
& \left|\psi^{ \pm}\right\rangle=N^{ \pm}\left(-\left(\frac{b \pm \xi}{J-i J_{z} D}\right)|01\rangle+|10\rangle\right), \\
& \left|\Sigma^{ \pm}\right\rangle=M^{ \pm}\left(-\left(\frac{B \pm \eta}{J \gamma}\right)|00\rangle+|11\rangle\right),
\end{aligned}
$$

and

$$
\begin{aligned}
& \varepsilon_{1,2}=-\frac{1}{2} J_{z} \pm \xi \\
& \varepsilon_{3,4}=\frac{1}{2} J_{z} \pm \eta .
\end{aligned}
$$

where

$$
\begin{aligned}
& \mu_{ \pm}=\frac{e^{-\frac{\beta J_{z}}{2}}}{Z}\left(\cosh \beta \eta \mp \frac{B}{\eta} \sinh \beta \eta\right) \\
& w_{1,2}=\frac{e^{\frac{\beta J_{z}}{2}}}{Z}\left(\cosh \beta \xi \mp \frac{b}{\xi} \sinh \beta \xi\right) \\
& \nu=-\frac{J \gamma e^{-\frac{\beta J_{z}}{2}}}{Z \eta} \sinh \beta \eta \\
& z=-\frac{\left(J+i J_{z} D\right) e^{\frac{\beta J_{z}}{2}}}{Z \xi} \sinh \beta \xi
\end{aligned}
$$

and $Z=2 e^{\frac{\beta J_{z}}{2}}\left(\cosh \beta \xi+e^{-\beta J_{z}} \cosh \beta \eta\right)$.

In what follows, our purpose is to quantify the amount of entanglement of the above two-qubit system versus the parameters of the system, with the main concerning on $D$. For density matrix in the form (8), one can show, by straight forward calculations, that the square roots of the eigenvalues of matrix $R=\rho \tilde{\rho}$ are:

$$
\begin{aligned}
\lambda_{1,2} & =\left|\sqrt{w_{1} w_{2}} \pm\right| z|| \\
& =\frac{e^{\frac{\beta J_{z}}{2}}}{\xi Z}\left|\sqrt{\xi^{2}+J^{2}+\left(J_{z} D\right)^{2} \sinh ^{2} \beta \xi} \pm \sqrt{J^{2}+\left(J_{z} D\right)^{2}} \sinh \beta \xi\right|, \\
\lambda_{3,4} & =\left|\sqrt{\mu_{+} \mu_{-}} \mp \nu\right| \\
& =\frac{e^{-\frac{\beta J_{z}}{2}}}{\eta Z}\left|\sqrt{\eta^{2}+(J \gamma)^{2} \sinh ^{2} \beta \eta} \mp J \gamma \sinh \beta \eta\right| .
\end{aligned}
$$




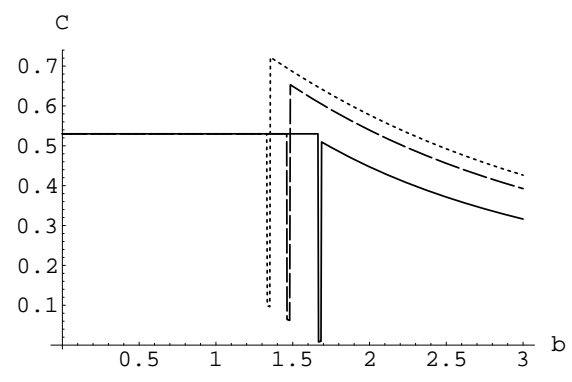

FIG. 1: The ground state concurrence vs. $b$ for $D=0$ (solid line), $D=0.8$ (dashed line) and $D=1$ (doted line) where $B=0.8, J=1, J_{z}=-1$ and $\gamma=0.5$.

Now, it is easy to calculate the concurrence. Without loss of generality we can assume $J>0$ and $\gamma>0$, since the above formula are invariant under substitution $J \rightarrow$ $-J$ and $\gamma \rightarrow-\gamma$. For the special case $D=0$, these equations give the same results as ref [17]. The obtained results are given in the following subsections.

\section{A. Ground state entanglement and critical parameters}

The behavior of the system at the quantum phase transition (QPT) point [49], such as $B=B_{c}, b=b_{c}$ or $D=D_{c}$, may be determined from the density matrix at $T=0$, at which the system is in its ground state. If we consider $T \rightarrow 0$ (or $\beta \rightarrow \infty$ ) then the concurrence $\mathrm{C}$ can be written analytically as:

$$
\begin{aligned}
& \text { if } \xi<\eta-J_{z} \\
& \text { if } \xi=\eta-J_{z} \\
& \text { if } \xi>\eta-J_{z}
\end{aligned}
$$

Also, this formula can be derived by calculating entanglement of ground state of $\mathrm{H}$ directly: When $\epsilon_{4}<\epsilon_{2}$ (or $\xi<\eta-J_{z}$ ) the ground state of $\mathrm{H}$ is $\left|\Sigma^{-}\right\rangle$and then $C=\left|\frac{J \gamma}{\eta}\right|$ and when $\epsilon_{2}<\epsilon_{4}$ (or $\left.\xi>\eta-J_{z}\right)$ the ground state of $\mathrm{H}$ is $\left|\psi^{-}\right\rangle$and then $C=\left|\frac{\sqrt{J^{2}+\left(J_{z} D\right)^{2}}}{\xi}\right|$. On the other hand, at critical point (where $\epsilon_{4}=\epsilon_{2}$ or $\xi<\eta-J_{z}$ ), the ground state of the system is an equally mixture of $\left|\Sigma^{-}\right\rangle$and $\left|\psi^{-}\right\rangle$i.e. $|G S\rangle=\left(\left|\Sigma^{-}\right\rangle+\left|\psi^{-}\right\rangle\right) / 2$ and then $C=\frac{1}{2}|| \frac{J \gamma}{\eta}\left|-\frac{\sqrt{J^{2}+\left(J_{z} D\right)^{2}}}{\xi}\right|$.

The concurrence $C$ as a function of $b$ (at $T=0)$ for three values of $D(D=0,0.8,1)$ are plotted in fig. 1. With increasing $b$, the concurrence $C$ is initially constant and equal to $C=\left|\frac{J \gamma}{\eta}\right|=0.53$, then drops suddenly at critical value of $\mathrm{b}\left(b_{c}=\sqrt{\left(\eta-J_{z}\right)^{2}-\left(\left(J_{z} D\right)^{2}+J^{2}\right)}\right)$. At this point $\left(T=0, b=b_{c}\right)$, the concurrence becomes a nonanalytical function of $b$ and QPT occurs [17]. For $b>b_{c}$, concurrence $C$ undergoes a revival before decreasing to zero. The amount of concurrence at revival region depends on $D$, by increasing $D$ the revival is greater. Furthermore, by increasing $D, b_{c}$ decreases i.e. for larger $D$, the critical point and hence revival phenomenon occurs in smaller $b$. The role of $D$ is more obvious in figs. 2a-2c where concurrence is plotted in terms of $D$. These figures show that, when $D$ reaches its critical value defined by

$$
D_{c}=\sqrt{\left.\left(\eta-J_{z}\right)^{2}-\left(b^{2}+J^{2}\right)\right)} /\left|J_{z}\right|,
$$

the concurrence drops and exhibit a revival phenomenon when $D$ crosses its critical value $D_{c}$. In the revival region (larger $D$ ) the concurrence reaches its maximum value $(C=1)$. Fig. 2a illustrates the ground state concurrence variation versus $D$ for three values of by decreasing $b$, the critical value of $D\left(D_{c}\right)$ increases. Fig. $2 \mathrm{~b}$ shows ground state concurrence versus $D$ for three values of $\gamma$, by increasing $\gamma, D_{c}$ increases (amount of $\gamma$ determines the value of entanglement before reaching the critical point). In fig. 2c the ground state concurrence versus $D$ is plotted for three values of $J_{z}$, when $\left|J_{z}\right|$ increases, $D_{c}$ also increases.

Hence, we can control value of $D_{c}$ by adjusting the parameters of the system such as $b, \gamma$ and $J_{z}$. We demand to decrease $D_{c}$ (because for $D>D_{c}$ concurrence will be maximized). Therefore we should choose, $\left|J_{z}\right|=1, \gamma$ small and $b$ as large as possible.

\section{B. Thermal entanglement}

Since the relative magnitude of $\lambda_{i}(i=1,2,3,4)$ depends on the parameters involved, they cannot be ordered by magnitude without knowing the values of the parameters. This prevents one from writing an analytical expression for concurrence. For particular parameters, $C$ can be evaluated numerically. The role of each parameter can be seen by fixing other parameters and drawing variation of entanglement for 

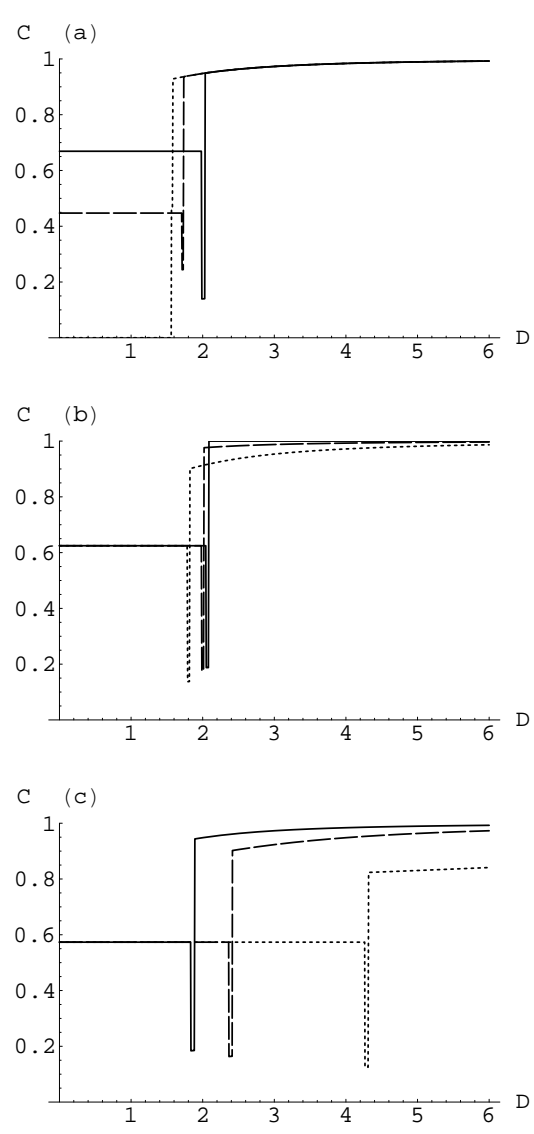

FIG. 2: The concurrence of the ground state vs. D. a) for $b=0$ (solid line), $b=0.5$ (dashed line) and $b=1$ (doted line), $J_{z}=-1$ and $\gamma=0.8 \mathrm{~b}$ ) for $\gamma=0.9$ (solid line), $\gamma=0.5$ (dashed line) and $\gamma=0$ (doted line), $J_{z}=-1$ and $b=0.75 \mathrm{c}$ ) for $J_{z}=-1$ (solid line), $J_{z}=0.5$ (dashed line) and $J_{z}=0.1$ (doted line), $b=0.75$ and $\gamma=0.7$.

specific values of that parameter. Cross influence of two parameters can be shown in 3D plots of entanglement. Thermal entanglement versus system's parameters is depicted in figures 3-5. The concurrence as a function of $T$ and $D$ is shown in fig. 3. The analysis of results of this figure are given in the following. Let $C_{j}^{\prime}=2 \lambda_{j}-\sum_{i=1}^{4} \lambda_{i}(j=1,2,3,4)$, it is evident that the function $C_{j} \stackrel{\max }{i}\left\{0, C_{j}^{\prime}\right\}$ is the concurrence of the system if and only if $\lambda_{j}=\lambda_{\max }=\max \left\{\lambda_{1}, \lambda_{4}\right\}$ (Since $J, \gamma>0$ we have $\lambda_{4}>\lambda_{3}$ and $\lambda_{1}>\lambda_{2}$ ). We can divide the regions of fig. 3 in four parts:

i) Region for which $T<T_{c 1}$ (see below) and $D<D_{c}$; in this region $\lambda_{\max }=\lambda_{4}$ and then $C_{4}^{\prime}=\lambda_{4}-\lambda_{3}-\lambda_{2}-\lambda_{1}$ determines the amount of entanglement. According to equation (10), the values of $\lambda_{4}$ depends on $\gamma$ and $\eta$ (or equivalently $\gamma$ and $B$ ). Thus in this region we can manipulate the amount of entanglement by adjusting $\gamma$ and
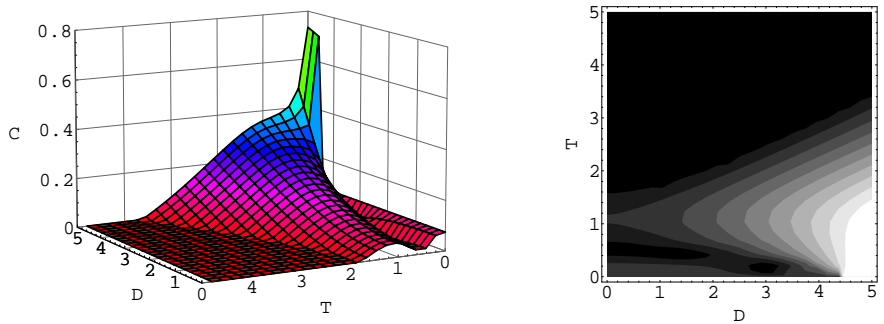

FIG. 3: color online) Thermal concurrence vs. $\mathrm{T}$ and D. Where $B=4, b=2.5, J=1$ and $\gamma=0.3$.
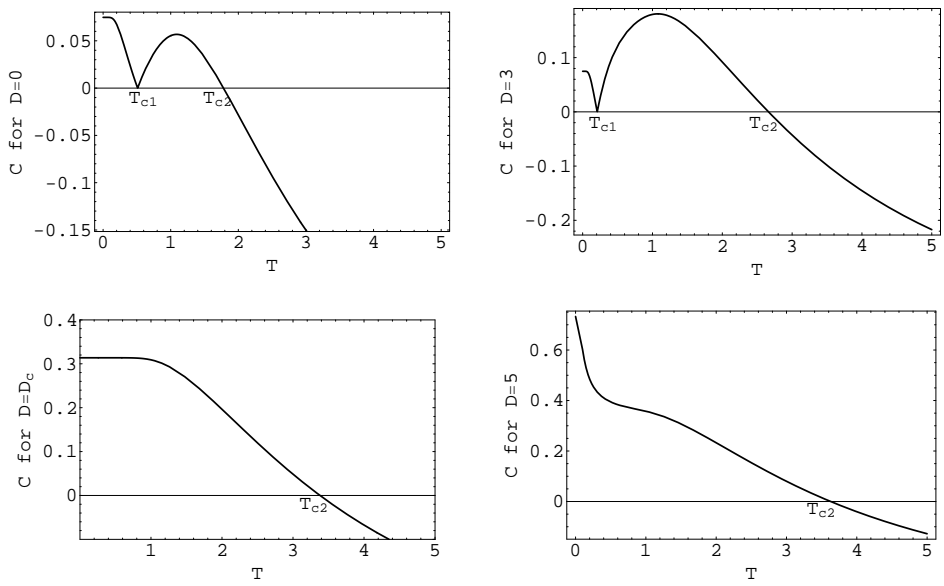

FIG. 4: Thermal concurrence vs. $T$ for different values of $D$. The parameters are the same as fig. 3 In this case $D_{c} \simeq 4.51$.

$B$. The first critical temperature $\left(T_{c 1}\right)$, is the point at which $\lambda_{\max }=\lambda_{4}=\lambda_{1}$ and hence $C=0$.

ii) Revival region, for which $T_{c 1}<T<T_{c 2}$ (see below) and $D<D_{c}$; in this region $\lambda_{\max }=\lambda_{1}$ and hence $C_{1}^{\prime}$ determines the amount of entanglement. According to equation (10), the value of $\lambda_{1}$ is adjustable by changing values of $D$ and $\xi$ (or equivalently $D$ and $b$ ) and hence the parameters $D$ and $b$ play an important role in quantifying the amount of entanglement, in this region. For $T>T_{c 1}$, the value of $\lambda_{1}$ and also the rate of enhancement of the function $\lambda_{1}$ with $T$ increases as $D$ increases. Enhancement of the function $\lambda_{1}$ with $T$, cause $C_{1}^{\prime}$ rise to a positive number and thus the entanglement undergoes a revival. Since the rate of enhancement of the function $\lambda_{1}$ with $T$ is an increasing function of $D$, the amount of revival increases as $D$ increases. When $T$ reaches the value $T=T_{c 2}$ (second critical temperature), $\lambda_{1}$ tend to zero again and thus the entanglement vanishes.

iii) Region for which $D>D_{c}$, for all values of $T$; in this region $\lambda_{\max }=\lambda_{1}$ and $C_{1}^{\prime}$ is the entanglement indicator. The maximum value of the entanglement occurs in this region. At zero temperature the entanglement has its maximum value and by increasing the temperature the 

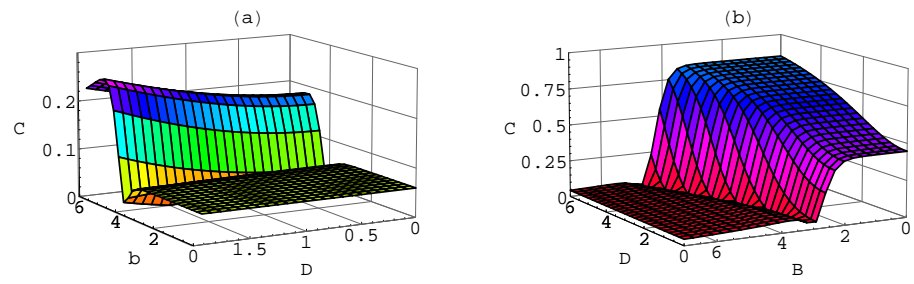

FIG. 5: (color online) (a) Thermal concurrence vs. b and D, where $\mathrm{B}=5$, (b) Thermal concurrence vs. $\mathrm{B}$ and $\mathrm{D}$, where $\mathrm{b}=2$. Here $T=0.3, J=1, J_{z}=0.5$ and $\gamma=0.3$

system loses its entanglement and ultimately vanishes at $T=T_{c 2}$. In this region no revival phenomenon occurs.

iv) Region for which $T \geq T_{c 2}$ for all values of $D$; in this region all values of $C_{j}^{\prime}(j=1,2,3,4)$ have negative values and then the entanglement is zero for all values of $D$ and the other parameters.

Notice that, $T_{c 1}$ and $T_{c 2}$ are sensitive functions of $D$. In fig. 4, we try to demonstrate these facts, by illustrating the function of thermal concurrence vs. $T$ for few values of $D$. This figure shows that for $D<D_{c}, T_{c 1}$ is a decreasing function of $D$ but for $D>D_{c}, T_{c 1}$ is undefined. In contrast, $T_{c 2}$ is an increasing function of $D$ for all values of $T$, i.e. we can create and maintain the nonzero entanglement at larger temperatures. In summary, fig. 3 shows that: For $T_{c 1}<T<T_{c 2}$ and $D<D_{c}$, there are regions in $D-T$ plane where increasing of temperature first increases the entanglement (revival region) and then tends to decrease the entanglement and ultimately for $T>T_{c 2}$ entanglement vanishes. The maximum entanglement exists at zero temperature and for large $D$. In the revival region and region for which $D>D_{c}$, increasing $D$ causes the entanglement to increase. In this region, $T_{c 1}$ decreases and $T_{c 2}$ increases as $D$ increases and hence the width of the revival region increases. In all regions $T_{c 2}$ is an increasing function of $D$, thus when $D$ is large enough, the entanglement can exists for larger temperatures. Furthermore, the parameter $D$ plays the role of parameter b. Fig. 5a, shows the variation of entanglement as a function of $b$ (inhomogeneity of magnetic field) and $D$. For fixed $D$, there are three region in this figure i) Main region where $b<b_{c}$; in this region entanglement is constant ii) Collapse region where $b=b_{c}$; in this region entanglement decreases suddenly iii) Revival region where $b>b_{c}$; in this region entanglement undergoes a revival. Amount of $D$ determines $b_{c}$ and hence edge of revival region. Furthermore, in the revival region increasing $D$ increases the entanglement. In fig. 5b, thermal entanglement is plotted vs. magnetic field $(B)$ and $D$. The role of $D$ is similar to its role in fig. 5a, enhancement of $D$ improves $B_{c}$ and increases amount of entanglement in the revival region.

One can use the above entangled two-qubit system for performance the teleportation protocols. The next section is spend to this subject.

\section{THERMAL ENTANGLEMENT TELEPORTATION}

For the entanglement teleportation of a whole twoqubit system, a thermal mixed state in Heisenberg spin chain can be considered as a general depolarizing channel. Now we consider Lee and Kim's two qubit teleportation protocol $\left(P_{1}\right)$, and use two copies of the above two-qubit thermal state, $\rho_{T} \otimes \rho_{T}$, as resource [42]. Similar to standard teleportation, entanglement teleportation for the mixed channel of an input entangled state is destroyed and its replica state appears at the remote place after applying a local measurement in the form of linear operators. We consider as input a two-qubit in the special pure state $\left|\psi_{i n}\right\rangle=\cos \theta / 2|10\rangle+e^{i \phi} \sin \theta / 2|01\rangle(0 \leq$ $\theta \leq \pi, 0 \leq \phi \leq 2 \pi)$. The density matrix related to $\left|\psi_{i n}\right\rangle$ is in the form:

$$
\rho_{i n}=\left(\begin{array}{cccc}
0 & 0 & 0 & 0 \\
0 & a & c & 0 \\
0 & c^{*} & b & 0 \\
0 & 0 & 0 & 0
\end{array}\right),
$$

where $a=\sin ^{2} \theta / 2, b=\cos ^{2} \theta / 2$ and $c=\frac{1}{2} e^{-i \phi} \sin \theta$. Therefore concurrence of initial state is $C_{i n}=2$ | $e^{i \phi} \sin \theta / 2 \cos \theta / 2 \mid=\sin \theta$. The output (replica) state $\rho_{\text {out }}$ can be obtained by applying joint measurement and local unitary transformation on input state $\rho_{i n}$. Thus the out put state is given by 41

$$
\rho_{\text {out }}=\sum_{\mu, \nu} p_{\mu \nu}\left(\sigma_{\mu} \otimes \sigma_{\nu}\right) \rho_{\text {in }}\left(\sigma_{\mu} \otimes \sigma_{\nu}\right),
$$

where $\mu, \nu=0, x, y, z \quad\left(\sigma^{0}=I\right), \quad p_{\mu \nu}=$ $\operatorname{tr}\left[E^{\mu} \rho_{\text {channel }}\right] \operatorname{tr}\left[E^{\nu} \rho_{\text {channel }}\right]$ such that $\sum_{\nu, \mu} p_{\mu \nu}=1$ and $\rho_{\text {channel }}$ represent the state of channel which used for teleportation. Here $E^{0}=\left|\Psi^{-}\right\rangle\left\langle\Psi^{-}\left|, E^{1}=\right| \Phi^{-}\right\rangle\left\langle\Phi^{-}\right|$, $E^{0}=\left|\Phi^{+}\right\rangle\left\langle\Phi^{+}\right|$and $E^{0}=\left|\Psi^{+}\right\rangle\left\langle\Psi^{+}\right|$where $\left|\Psi^{ \pm}\right\rangle=$ $\frac{(|01\rangle \pm|10\rangle)}{\sqrt{2}}$ and $\left|\Phi^{ \pm}\right\rangle=\frac{(|00\rangle \pm|11\rangle)}{\sqrt{2}}$ are the Bell states.

By considering the two-qubit spin system as a quantum channel, the state of channel is $\rho_{\text {channel }}=\rho_{T}$ given in the equation (8) and hence one can obtain $\rho_{\text {out }}$ as

$$
\rho_{\text {out }}=\left(\begin{array}{cccc}
\alpha & 0 & 0 & \kappa \\
0 & a^{\prime} & c^{\prime} & 0 \\
0 & c^{\prime *} & b^{\prime} & 0 \\
\kappa & 0 & 0 & \alpha
\end{array}\right),
$$

where

$$
\begin{aligned}
& \alpha=\left(w_{1}+w_{2}\right)\left(\mu^{+}+\mu^{-}\right), \\
& \kappa=4 \operatorname{Re}[z] \nu \cos \phi \sin \theta \\
& a^{\prime}=\left(\mu^{+}+\mu^{-}\right)^{2} \cos ^{2} \frac{\theta}{2}+\left(w_{1}+w_{2}\right)^{2} \sin ^{2} \frac{\theta}{2} \\
& b^{\prime}=\left(w_{1}+w_{2}\right)^{2} \cos ^{2} \frac{\theta}{2}+\left(\mu^{+}+\mu^{-}\right)^{2} \sin ^{2} \frac{\theta}{2} \\
& c^{\prime}=2 e^{-i \phi}\left((\operatorname{Re}[z])^{2}+e^{2 i \phi} \nu^{2}\right) \sin \theta .
\end{aligned}
$$


Following the above we can determine concurrence of out put state by calculating positive square roots of $R_{\text {out }}=$ $\rho_{\text {out }} \tilde{\rho}_{\text {out }}$, i.e. $\lambda_{i}^{\prime}$ s. It is easy to show

$$
\begin{aligned}
\lambda_{1,2}^{\prime}= & \mid \frac{\sqrt{C_{i n}^{2}\left(\cosh ^{2} \beta \eta-e^{2 \beta J_{z}} \cosh ^{2} \beta \xi\right)^{2}+4 e^{2 \beta J_{z}} \cosh ^{2} \beta \eta \cosh ^{2} \beta \xi}}{2\left(\cosh \beta \eta+e^{\beta J_{z}} \cosh \beta \xi\right)^{2}} \\
& \pm \frac{\left|C_{i n}\left(\left(\frac{J \gamma}{\eta}\right)^{2} e^{2 i \phi} \sinh ^{2} \beta \eta+\left(\frac{J}{\xi}\right)^{2} e^{2 \beta J_{z}} \sinh ^{2} \beta \xi\right)\right|}{2\left(\cosh \beta \eta+e^{\beta J_{z}} \cosh \beta \xi\right)^{2}} \mid, \\
\lambda_{3,4}^{\prime}= & \left|\frac{\cosh \beta \eta \cosh \beta \xi \pm C_{i n}\left(\frac{J \gamma}{\eta}\right)\left(\frac{J}{\xi}\right) \sinh \beta \eta \sinh \beta \xi \cos \phi}{\left(\cosh \beta \eta+e^{\beta J_{z}} \cosh \beta \xi\right)^{2}}\right| e^{\beta J_{z}} .
\end{aligned}
$$

Thus $C_{\text {out }}=C\left(\rho_{\text {out }}\right)=\max \left\{0,2 \lambda_{\max }^{\prime}-\sum_{i=1}^{4} \lambda_{i}^{\prime}\right\}$ is computable when the parameters of channel are known. The function $C_{\text {out }}$ is dependent on the entanglement of initial state and the parameters of the channel (which determine the entanglement of channel). The $C_{\text {out }}$ is nonzero only for particular choice of channel's parameters for which $C_{\text {channel }}$ is greater than a critical value. The figs. 6 and 8 depict behavior of $C_{\text {out }}$ versus the parameters of the channel and $C_{i n}$. For the case $J_{z}>0$, the entanglement of replica state $\left(C_{\text {out }}\right)$ increases linearly as $C_{i n}$ increases. The rate of this enhancement is determined by $D$ (indeed, by $C_{\text {channel }}(D)$ ). But for the case $J_{z}<0, C_{\text {out }}$ is zero for small values of $D$. As $D$ crosses a threshold value, $C_{\text {out }}$ increase when $C_{i n}$ increase with the rate determined by amount of $D$ (equivalently $C_{\text {channel }}(D)$ ). The figs. $6 \mathrm{~b}$ and $6 \mathrm{c}$ show that the parameter of inhomogeneity $(b)$ can plays role of $D$.

- The Fidelity of entanglement teleportation: Fidelity between $\rho_{\text {in }}$ and $\rho_{\text {out }}$ characterizes the quality of teleported state $\rho_{\text {out }}$. When the input state is a pure state, we can apply the concept of fidelity as a useful indicator of teleportation performance of a quantum channel [43, 48]. The maximum fidelity of $\rho_{\text {in }}$ and $\rho_{\text {out }}$ is defined to be

$$
\begin{aligned}
F\left(\rho_{\text {in }}, \rho_{\text {out }}\right) & =\left\{\operatorname{Tr}\left[\sqrt{\left(\rho_{\text {in }}\right)^{\frac{1}{2}} \rho_{\text {out }}\left(\rho_{\text {in }}\right)^{\frac{1}{2}}}\right]\right\}^{2} \\
& =\left\langle\psi_{\text {in }}\left|\rho_{\text {out }}\right| \psi_{\text {in }}\right\rangle .
\end{aligned}
$$

By substituting $\rho_{\text {in }}$ and $\rho_{\text {out }}$ from above, we have

$$
F\left(\rho_{\text {in }}, \rho_{\text {out }}\right)=a^{\prime} \sin ^{2} \frac{\theta}{2}+b^{\prime} \cos ^{2} \frac{\theta}{2}+\operatorname{Re}\left[c^{\prime} e^{-i \phi}\right] \sin \theta(19)
$$

simplifying the above formula we find that the maximum fidelity $F\left(\rho_{\text {in }}, \rho_{\text {out }}\right)$ depends on initial entanglement $\left(C_{i n}\right)$ :

$$
F\left(\rho_{\text {in }}, \rho_{\text {out }}\right)=f^{c}+f^{q} C_{\text {in }}^{2},
$$
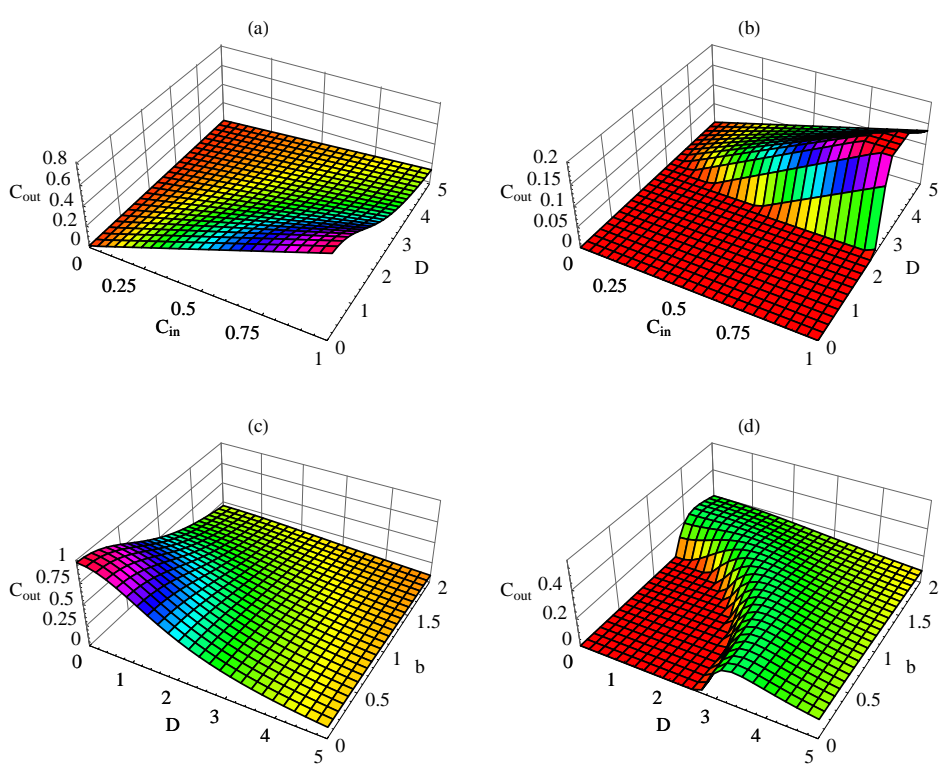

FIG. 6: (color online) Entanglement of output state $\left(C_{\text {out }}\right)$ vs. the channel's parameters and $C_{i n} . C_{o u t}$ vs. $D$ and $C_{i n}$ for a) $b=0.5$ and $J_{z}>0$ and b) $b=0.5$ and $J_{z}<0$. Also, $C_{\text {out }}$ vs. $b$ and $D$ for c) $C_{i n}=1$ and $J_{z}>0$ and d) $C_{i n}=1$ and $J_{z}<0$. Where $J=1, T=0.1, B=1$ and $\gamma=0.3$.

where $f^{c}=\left(w_{1}+w_{2}\right)^{2}$ and $f^{q}=\frac{1}{2}-\left(w_{1}+w_{2}\right)+$ $2\left(\nu^{2} \cos 2 \phi+(\operatorname{Re}[z])^{2}\right)$. The functions $f^{c}$ and $f^{q}$ are dependent on the channel's parameters only (we consider $\phi=0)$ and hence are relate to the entanglement of the channel. This formula is the same as the results of ref. [42] [50], but in spite of the werner states, $f^{q}$ can be a positive number for Heisenberg chains. This means that, there exists a channel such that it can teleport more entangled initial state with more fidelity! but it is not a useful claim because, when we choose the parameters of the channel such that $f^{q}>0$ then $f^{c}$ decreases and ultimately $F\left(\rho_{\text {in }}, \rho_{\text {out }}\right)$ becomes smaller than $\frac{2}{3}$ which means 

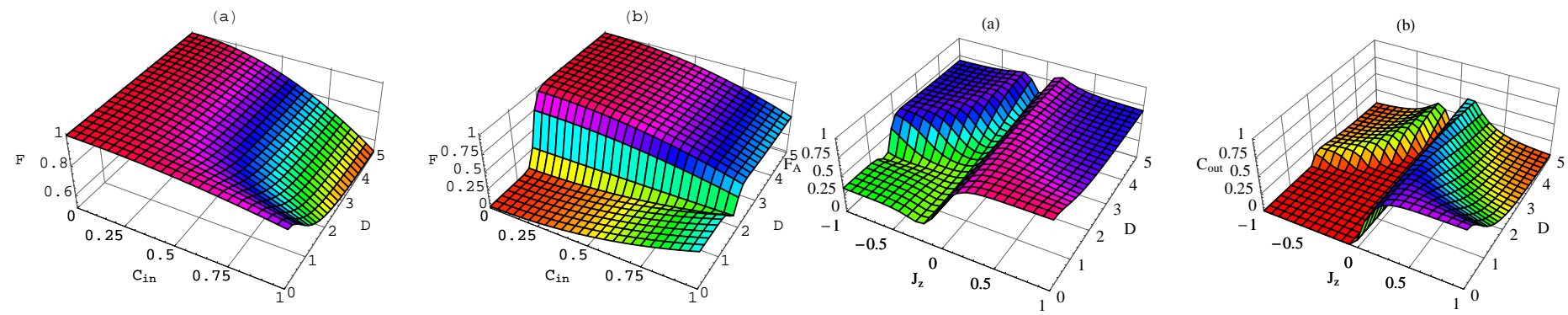

FIG. 7: (color online) $F\left(\rho_{\text {in }}, \rho_{\text {out }}\right)$ vs. $C_{\text {in }}$ and $D$ for a) $J_{z}>0$ and b) $J_{z}<0$. Where $J=1, T=0.1, B=1, b=0.5$ and $\gamma=0.3$

that the entanglement teleportation of mixed state is inferior to classical communication. Thus, to obtain the same proper fidelity, the larger entangled channel are required for larger entangled initial state. The fig. 7 emphasize the above notes.

The average fidelity $F_{A}$ is another useful concept for characterizing the quality of teleportation. The Average fidelity $F_{A}$ of teleportation can be obtained by averaging $F\left(\rho_{\text {in }}, \rho_{\text {out }}\right)$ over all possible initial states:

$$
F_{A}=\frac{\int_{0}^{2 \pi} d \phi \int_{0}^{\pi} F\left(\rho_{\text {out }}, \rho_{\text {in }}\right) \sin \theta d \theta}{4 \pi},
$$

and for our model $F_{A}$ can be written as

$$
F_{A}=\frac{\xi^{2} \cosh ^{2} \beta \eta+e^{2 \beta J_{z}}\left(2 \xi^{2} \cosh ^{2} \beta \xi+J^{2} \sinh ^{2} \beta \xi\right)}{3 \xi^{2}\left(\cosh \beta \eta+e^{\beta J_{z}} \cosh \beta \xi\right)^{2}}
$$

In the case of an isotropic XXX Heisenberg chain in the absence of magnetic field with spin-orbit interaction, this equation gives the same as results of ref. 445. The function $F_{A}$ is dependent on the channel's parameters. The fig. 8 gives a plot of $F_{A}, C_{\text {out }}, F\left(\rho_{\text {in }}, \rho_{\text {out }}\right)$ and $C_{\text {channel }}$ versus the channel's parameters. This figure shows that, in the case of $J_{z}>0, F_{A}, F\left(\rho_{\text {in }}, \rho_{\text {out }}\right)$ and $C_{\text {out }}$ decrease when $D$ increases. In this case $F_{A}$ approaches $\frac{2}{3}$ for large values of $D$.

In contrast, in the case of $J_{z}<0$ and for small values of $D, F_{A}$ and $F\left(\rho_{\text {in }}, \rho_{\text {out }}\right)$ has a constant value (smaller than $\frac{2}{3}$ ) and $C_{\text {out }}$ is zero. As $D$ becomes larger than a threshold value, $C_{\text {channel }}$ undergoes a revival and then decreases for larger values of $D$. Since in the revival region, $C_{\text {channel }}$ has its maximum value, $F_{A}, F\left(\rho_{\text {in }}, \rho_{\text {out }}\right)$ and $C_{\text {out }}$ increase in this region such that for particular interval of $D, F_{A}$ becomes larger than $\frac{2}{3}$ and ultimately, tends to $\frac{2}{3}$ for larger $D$. In summary, the fidelity of teleportation and entanglement of replica state are dependent on the entanglement of channel which is tunable by the channel's parameters (such as $D, b, B, \ldots$ ). The effect of D is more desirable in the case of $J_{z}<0$. In this case for a certain values of $D, F_{A}$ becomes greater than $\frac{2}{3}$, this make the channel useful for performance the teleportation protocol. For large $D, F_{A}$ tends to $\frac{2}{3}$.
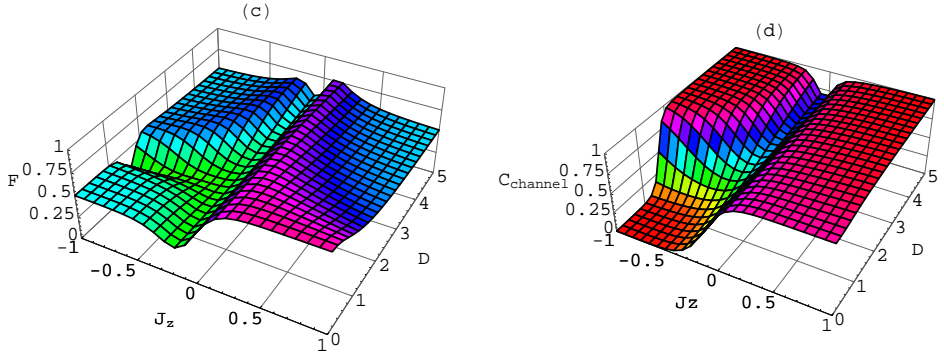

FIG. 8: (color online) a) Average fidelity (a), Output entanglement (b), Fidelity between $\rho_{\text {in }}$ and $\rho_{\text {out }}$ (c) and the entanglement (d) of channel versus $J_{z}$ and $D$. Where $J=1, T=$ $0.1, B=1, b=0.5, \gamma=0.3$ and $C_{i n}=1$

\section{DISCUSSION}

The entanglement of a two-qubit XYZ Heisenberg system in the presence of an inhomogeneous magnetic field and spin-orbit interaction is investigated. By turning on the spin-orbit interaction we can change the behavior of the system without manipulating the other parameters. We have shown that the critical values of $B, b$ and $T$ are adjustable by $D$ and we can improve the critical values of $B, b$ and T. Increasing $D$ cause to volume enhancement of revival region and also enhancement of entanglement in the revival region. Also, entanglement teleportation via two copies of above two-qubit system is studied. We have shown that, by introducing SO interaction, the entanglement of replica state and fidelity can be increased for the case of $J_{z}<0$, in spite to the case $J_{z}>0$. When $D$ becomes very large, the fidelity approaches $\frac{2}{3}$, which is the maximal value for the classical communication. 
[1] A. Einstein, B. Podolsky and N. Rosen, Phys. Rev. 47, 777 (1935)

[2] E. Schrödinger, Naturwiss. 23, 807 (1935)

[3] M. A. Neilsen and I. L. Chuang, Quantum computation and quantum information (Cambridge University Press) (fifth printing 2004)

[4] J. Audretsch, Entangled system, WILEY-VCH Verlag (2007)

[5] N. Canosa and R. Rossignoli, Phys. Rev. A 69, 052306 (2004)

[6] S. Hill and W. K. Wooters, Phys. Rev. Lett. 78 (1997; W. K. Wootres, Phys. Rev. Letts., 80, 2245 (1998)

[7] J. Eisert, Entanglement in Information Theory, Ph.D thesis (Februray 2001)

[8] M. A. Nielsen, e-print quant-ph/0011036

[9] D. Gunlycke, V. M. Kendon, V. Vedral, Phys. Rev. A 64, $042302(2001)$

[10] M. C. Arnesen, S. Bose, V. Vedral, Phys. Rev. Lett. 87, 017901 (2001)

[11] G. Lagmago Kamta and Anthony F. Starace, Phys. Rev. Lett., 88, 107901 (2002)

[12] Y. Sun, Y. chen and H. Chen, Phys. Rev. A 68, 044301 (2003)

[13] G. F. Zhang and S. Li, Phys. Rev. A 72, 034302 (2005)

[14] M. Asoudeh and V. Karimipour, Phys. Rev. A 71, 022308 (2005)

[15] L. Zhou, H. S. Song, Y. Q. Guo and C. Li, Phys. Rev. A 68, 024301 (2003)

[16] G. Rigolin, Int. J. Quant. Inf. 2, 393 (2004)

[17] G. H. Yang, W. B. Gao, L. Zhou and H.S. Song, quant-ph/0602051 (2006)

[18] I. Dzyaloshinski, J. Phys. Chem. Solids 4, 241 (1958)

[19] T. Moriya, Phys. Rev. 117, 635 (1960)

[20] T. Moriya, Phys. Rev. Lett.4, 228 (1960)

[21] T. Moriya, Phys. Rev. 120, 91 (1960)

[22] N. E. Bonesteel and D. Stepaneko, Phys. Rev. Lett. 87, 207901 (2001)

[23] G. Burkard and D. Loss, Phys. Rev. Lett. 88, 047903 (2002)

[24] L. A. Wu and D. Lidar, Phys. Rev. A 66,062314 (2002)

[25] X. Wang, Phys. Lett. A 281, 101 (2001)

[26] D. Loss and D.P. Divincenzo, Phys. Rev. A 57, 120 (1998)

[27] D. DiVincenzo, Phys. Rev. A 51,1015 (1995)

[28] W. A. Coish and D. Loss, e-print cond-mat/0606550 (1998) and G. Burkard, D. Loss and D. P. Divincenzo , Phys. Rev. B 59, 2070 (2002)

[29] H. Xiong, Coherent-Induced Entanglement, Ph.D thesis
(May 2006)

[30] D. Cory, A. Fahmy and T. Havel, Proc. Natl. Acad. Sci. USA 94,1634 (1997)

[31] I. L. Chuang, N. A. Gershenfeld and M. Kubinec, Phys. Rev. Lett. 80,3408 (1998)

[32] J. A. Jones, M. Mosca and R. H. Hansen, Nature (London) 393, 344 (1998)

[33] A. Pfund, K. Ensslin and R. Leturcq, Phys. Rev. B 76, 161308(R) (2007)

[34] N. Zhao, L. Zhong, J. L. Zhu and C. P. Sun, Phys. Rev. A 74, 075307 (2006)

[35] S. Debald and C. Emary, Phys. Rev. Lett. 94, 226803 (2005)

[36] V. N. Golovach, M. Borhani, and D. Loss, Phys. Rev. B 74, 165319 (2006); D. V. Bulaev and D. Loss, Phys. Rev. Lett. 98, 097202 (2007)

[37] C. Flindt, A. S. Srensen, and K. Flensberg, Phys. Rev. Lett. 97, 240501 (2006)

[38] C. H. Bennett, G. Brassard, C. Crepeau, R. Josza, A. Peres and W. K. Wooters, Phys. Rev. Letts. 70, 18951899 (1993)

[39] S. Popescu, Phys. Rev. Letts. 72, 797-799 (1994)

[40] M. Horodecki, P. Horodecki and R. Horodecki, Phys. Rev A 60, 1888 (1999)

[41] G. Bowen and S. Bose, Phys. Rev. Lett. 87, 2679011

[42] J. Lee, M. S. Kim, Phys. Rev. Letts. 84,4236 (2000)

[43] Y. Yeo, Phys. Rev. A 66, 062312 (2002) and Y. Yeo, quant-ph/023014 (2002)

[44] Y. Yeo, T. Liu, Y. Lu and Q. Yang, J. Phys. A: Math. Gen. 38, 3235 (2005)

[45] G. F. Zhang, Phys. Rev. A 75, 034304 (2007)

[46] S. Olav Skrøvseth, e-print quant-ph/0612133 (2006)

[47] S. Sachdiv, Quantum phase transition (Cambridge University Press, Cambridge, UK,1999)

[48] R. Josza, J. mod. Opt. 41, 2315, (1994)

[49] Hint: A phase transition can, strictly speaking, only occur in the thermodynamic limit of $\sim 10^{23}$ particles. However, one may see traces of what may become a phase transition at smaller systems, it is important to emphasize that no actual phase transition can occur in this system [46, 47].

[50] In the ref. 42], Lee and Kim use the negativity as a measure of entanglement and the werner states as resource. In $2 \times 2$ systems, the negativity coincides with concurrence for pure state and Werner states [7] 